\title{
EQUIVARIANCE AND IMPRIMIVITY FOR DISCRETE HOPF $C^{*}$-COACTIONS
}

\section{S. KALISZEWSKI AND JOHN QUIGG}

Let $U, V$, and $W$ be multiplicative unitaries coming from discrete Kac systems such that $W$ is an amenable normal submultiplicative unitary of $V$ with quotient $U$. We define notions for right-Hilbert bimodules of coactions of $S_{V}$ and $\widehat{S}_{V}$, their restrictions to $S_{W}$ and $\widehat{S}_{U}$, their dual coactions, and their full and reduced crossed products. If $N(A)$ denotes the imprimitivity bimodule associated to a coaction $\delta$ of $S_{V}$ on a $C^{*}$. algebra $A$ by $\mathrm{Ng}$ 's imprimitivity theorem, we prove that for a suitably nondegenerate injective right-Hilbert bimodule coaction of $S_{V}$ on ${ }_{A} X_{B}$, the balanced tensor products $N(A) \otimes_{A \times \widehat{S}_{W}}\left({ }_{A} X_{B} \times \widehat{S}_{W}\right)$ and $\left({ }_{A} X_{B} \times \widehat{S}_{V} \times{ }_{r} S_{U}\right) \otimes_{B \times \widehat{S}_{V} \times_{r} S_{U}} N(B)$ are isomorphic right-Hilbert $A \times \widehat{S}_{V} \times{ }_{r} S_{U}-B \times \widehat{S}_{W}$ bimodules. This can be interpreted as a natural equivalence between certain crossed-product functors.

\section{INTRODUCTION}

Since Baaj and Skandalis introduced multiplicative unitaries in [2] as a generalisation of locally compact groups, and proved a duality theorem [2, Théorème 7.5] for crossed products by coactions of the associated Hopf $C^{*}$-algebras, there has been much interest in extending other results for group actions and coactions to this context. Recently $\mathrm{Ng}$ [18] has defined notions of sub- and quotient multiplicative unitaries, and has proved that for multiplicative unitaries $U, V$, and $W$ coming from discrete Kac systems such that $W$ is an amenable normal submultiplicative unitary of $V$ with quotient $U$, and for any injective nondegenerate coaction $\delta$ of $S_{V}$ on a $C^{*}$-algebra $A$, the iterated crossed product $A \times_{\delta} \widehat{S}_{V} \times_{\widehat{\delta} \mid, r} S_{U}$ is Morita equivalent to $A \times_{\delta \mid} \widehat{S}_{W}[18$, Theorem 3.4]. This is an analog both of Green's celebrated imprimitivity theorem [9], which implies that for an action $\alpha$ of a group $G$ on $A$ and a closed normal subgroup $N$ of $G, A \times_{\alpha} G \times_{\widehat{\alpha} \mid} G / N$ is Morita equivalent to $A \times_{\alpha \mid} N$, and of Mansfield's imprimitivity theorem [15] for coactions (as

Received 5th January, 2000

This research was partially supported by the Australian Research Council and by the National Science Foundation (under Grant No. DMS9401253) and by the Office of the Vice Provost for Research at Arizona State University.

A substantial amount of this research was conducted while the first author visited the second at Arizona State University, and on another occasion while both authors visited the University of Newcastle. We particularly thank Iain Raeburn in Newcastle for his hospitality.

Copyright Clearance Centre, Inc. Serial-fee code: 0004-9727/00 \$A2.00+0.00. 
generalised to non-amenable groups in [11]), which provides a Morita equivalence between $A \times{ }_{\delta} G \times \times_{\bar{\delta} \mid, r} N$ and $A \times{ }_{\delta \mid} G / N$ for a coaction $\delta$ (satisfying a mild condition) of $G$ on $A$ and any closed normal subgroup $N$ of $G$. For discrete multiplicative unitaries, $\mathrm{Ng}$ 's theorem generalises Baaj-Skandalis duality (ignoring differences between full and reduced crossed products) in the same way that Green's theorem generalises Imai-Takai-Takesaki duality [10], and Mansfield's theorem generalises the duality of Katayama [13].

Now the significance of Green's theorem is that his imprimitivity bimodule may be viewed as a Hilbert $A \times_{\alpha \mid} N$-module with a nondegenerate left action of $A \times_{\alpha} G$ by adjointable operators, and thus allows induction of representations from $A \times_{\alpha \mid} N$ to $A \times_{\alpha} G$ via Rieffel's framework [21]. Similarly, Mansfield's bimodule allows induction of representations from $A \times_{\delta \mid} G / N$ to $A \times_{\delta} G$. The representation-inducing processes arising from these bimodules, and their interactions with one another, have received much attention lately (see $[3,8,5,12,20]$ ), and the method that has evolved is to work with the bimodules that implement the inducing maps on representations, rather than with those inducing maps themselves. We call the bimodules involved right-Hilbert bimodules; they are essentially imprimitivity bimodules ${ }_{K} X_{B}$ together with nondegenerate homomorphisms of $A$ into $M(K)$.

The equivariant right-Hilbert bimodules - that is, those right-Hilbert bimodules ${ }_{A} X_{B}$ which carry compatible actions or coactions of a group $G$ - turn out to be closely related to imprimitivity theorems. In work with Echterhoff and Raeburn which is currently in preparation we have shown, for example, that Green's imprimitivity theorem can be viewed as a natural equivalence between the crossed product functors $(A, G, \alpha) \mapsto A \times_{\alpha} G \times_{\widehat{\alpha} \mid} G / N$ and $(A, G, \alpha) \mapsto A \times_{\alpha \mid} N$ defined on a category whose objects are $C^{*}$-algebras with actions of $G$ and whose morphisms $(A, \alpha) \rightarrow(B, \beta)$ are (isomorphism classes) of equivariant right-Hilbert $A-B$ bimodules [4].

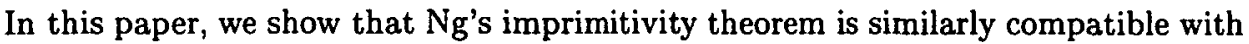
equivariant right-Hilbert bimodules. To do so, we must first develop a theory of coactions of Hopf $C^{*}$-algebras $S_{V}$ and $\widehat{S}_{V}$ on right-Hilbert bimodules, and their crossed products; this is done as efficiently as possible in Section 2 by building for the most part on Ng's imprimitivity bimodule apparatus [16]. In Section 3, we review Ng's fixed-point theorem [18, Proposition 2.11], since it provides the construction of the bimodule which appears in his imprimitivity theorem. Here we prove two lemmas relating $\mathrm{Ng}$ 's bimodule to the linking algebra and standard right-Hilbert bimodule (see below) constructions we use in proving our main theorem.

In the final section, we prove our main result: for $U, V$, and $W$ as in $\mathrm{Ng}$ 's theorem, and for a suitably nondegenerate injective right-Hilbert bimodule coaction of $S_{V}$ on ${ }_{A} X_{B}$,

$$
N(A) \otimes_{A \times \widehat{S}_{W}} X \times \widehat{S}_{W} \cong X \times \widehat{S}_{V} \times{ }_{r} S_{U} \otimes_{B \times \widehat{S}_{V} \times S_{r}} N(B)
$$

as right-Hilbert $A \times \widehat{S}_{V} \times{ }_{r} S_{U}-B \times \widehat{S}_{W}$ bimodules, where $N(A)$ denotes $\mathrm{Ng}$ 's $A \times \widehat{S}_{V} \times_{\mathrm{r}} S_{U}$ 
- $A \times \widehat{S}_{W}$ imprimitivity bimodule (and similarly for $N(B)$ ). As discussed above for group actions, this should give a natural equivalence between certain crossed-product functors, although we don't formalise this in the present paper. (Part of our point here is that any reasonable imprimitivity theorem should be compatible with equivariant rightHilbert bimodules, and that the proof of this, following the same strategy we use in the proof of Theorem 4.1, should be relatively straightforward.) Our theorem should have implications for induced and restricted representations of crossed products by Hopf $C^{*}$-algebras, and for equivariant $K K$-theory as in [1].

\section{Preliminaries}

For compatibility with Ng's work on imprimitivity bimodules, we define a rightHilbert $A-B$ bimodule over $C^{*}$-algebras $A$ and $B$ to be an imprimitivity bimodule ${ }_{K} X_{B}$ together with a nondegenerate homomorphism of $A$ into $M(K)$. If $X$ is a full Hilbert $B$-module (see [14]), then $X$ is a $\mathcal{K}_{B}(X)-B$ imprimitivity bimodule, and $M\left(\mathcal{K}_{B}(X)\right)=\mathcal{L}_{B}(X)$, so this is the same as having a nondegenerate action of $A$ by adjointable operators on $X$. Note that $K$ itself becomes a right-Hilbert $A-K$ bimodule by using the natural $K-K$ imprimitivity bimodule structure on $K$; we call this a standard right-Hilbert bimodule. We have the decomposition ${ }_{A} X_{B} \cong_{A} K \otimes_{K} X_{B}$ of any right-Hilbert bimodule as a balanced tensor product of a standard bimodule and an imprimitivity bimodule. We use the conventions of [6] regarding multiplier bimodules, linking algebras, and homomorphisms of imprimitivity bimodules.

Let $\left(S, \delta_{S}\right)$ be a Hopf $C^{*}$-algebra, and let $\delta: A \rightarrow M(A \otimes S)$ be a coaction of $S$ on a $C^{*}$-algebra $A$, as in [2, Definition 0.2]. The coaction $\delta$ is called nondegenerate if $\overline{\operatorname{span}}\left\{\delta_{A}(A)(1 \otimes S)\right\}=A \otimes S$. A covariant pair for $(A, S, \delta)$ on a $C^{*}$-algebra $B$ consists of a nondegenerate homomorphism $\theta: A \rightarrow M(B)$ and a unitary corepresentation $u \in M(B \otimes S)$ of $S$ such that

$$
(\theta \otimes \mathrm{id}) \circ \delta(a)=\operatorname{Ad}(u)(\theta(a) \otimes 1)
$$

for each $a \in A\left[17\right.$, Definition 2.8]. The full crossed product for $(A, S, \delta)$ is a $C^{*}$-algebra $A \times_{\delta} \widehat{S}$ together with a universal covariant pair $(j, v)$ for $(A, S, \delta)$ on $A \times_{\delta} \widehat{S}$ [17, Definition 2.11(b)]. If $S=\widehat{S}_{V}$ for a multiplicative unitary $V$ coming from a Kac system (see below), we write $A \times_{\delta} S_{V}$ (with no hat) for $A \times_{\delta} \widehat{S}$.

Let $V \in \mathcal{L}(H \otimes H)$ be a regular multiplicative unitary as in [2]. We let $L$ and $\rho$ denote the maps of $\mathcal{L}(H)$. into $\mathcal{L}(H)$ defined by

$$
L(\omega)=(\omega \otimes \mathrm{id})(V) \quad \text { and } \quad \rho(\omega)=(\mathrm{id} \otimes \omega)(V) ;
$$

then we have the associated reduced Hopf $C^{*}$-algebras

$$
S_{V}=\overline{\operatorname{span}}\left\{L(\omega) \mid \omega \in \mathcal{L}(H)_{*}\right\} \quad \text { and } \quad \widehat{S}_{V}=\overline{\operatorname{span}}\left\{\rho(\omega) \mid \omega \in \mathcal{L}(H)_{*}\right\}
$$


with comultiplications $\delta_{V}$ and $\widehat{\delta}_{V}$, respectively, given by

$$
\delta_{V}(x)=V(x \otimes 1) V^{*} \quad \text { and } \quad \widehat{\delta}_{V}(y)=V^{*}(1 \otimes y) V
$$

[2, Théorème 3.8]. The corresponding full Hopf $C^{*}$-algebras are denoted $\left(S_{V}\right)_{p}$ and $\left(\widehat{S}_{V}\right)_{p}$, but their comultiplications are still denoted $\delta_{V}$ and $\widehat{\delta}_{V}[2$, Corollaire A.6]. We view $L$ both as a faithful representation of $S_{V}$ and as a nondegenerate representation of $\left(S_{V}\right)_{p}$ on $\mathcal{L}(H)$; similarly, we view $\rho$ as a map on both $\widehat{S}_{V}$ and $\left(\widehat{S}_{V}\right)_{p}$ (see $[17$, Proposition 1.16(i)]).

The unitary corepresentations $u \in M\left(B \otimes S_{V}\right)$ of $S_{V}$ are in bijective correspondence with the nondegenerate homomorphisms $\nu:\left(\widehat{S}_{V}\right)_{p} \rightarrow M(B)\left[\mathbf{1 7}\right.$, Lemma2.6]. If $\left(A, S_{V}, \delta\right)$ is a coaction, by $[17$, Remark $2.12(\mathrm{~b})]$ we have

$$
A \times_{\delta} \widehat{S}_{V}=\overline{\operatorname{span}}\left\{j(a) \mu(y) \mid a \in A, y \in\left(\widehat{S}_{V}\right)_{p}\right\},
$$

where $\mu:\left(\widehat{S}_{V}\right)_{p} \rightarrow M\left(A \times_{\delta} \widehat{S}_{V}\right)$ is the nondegenerate homomorphism corresponding to $v$. For every pair of homomorphisms $\theta: A \rightarrow M(B)$ and $\nu:\left(\widehat{S}_{V}\right)_{p} \rightarrow M(B)$ coming from a covariant pair $(\theta, u)$, there is (by definition of the crossed product) a unique nondegenerate homomorphism $\theta \times \nu: A \times_{\delta} \widehat{S}_{V} \rightarrow M(B)$ such that

$$
(\theta \times \nu) \circ j=\theta \quad \text { and } \quad(\theta \times \nu) \circ \mu=\nu
$$

and the latter condition is equivalent to

$$
((\theta \times \nu) \otimes \mathrm{id})(v)=u
$$

Let $\pi_{L}=(\mathrm{id} \otimes L) \circ \delta: A \rightarrow \mathcal{L}_{A}(A \otimes H)$. Then the reduced crossed product [17, Definition 2.11(a)] is

$$
A \times_{\delta, r} \widehat{S}_{V}=C^{*}\left(\left\{\pi_{L}(a)(1 \otimes \rho(\omega)) \mid a \in A, \omega \in \mathcal{L}(H)_{*}\right\}\right) \subseteq \mathcal{L}_{A}(A \otimes H)
$$

The reduced crossed product by a coaction $\delta_{p}$ of $\left(S_{V}\right)_{p}$ is defined similarly, and we have

$$
A \times_{\delta_{p}, r}\left(\widehat{S}_{V}\right)_{p} \cong A \times_{\delta, r} \widehat{S}_{V}
$$

where the coaction $\delta=(\mathrm{id} \otimes L) \circ \delta_{p}$ of $S_{V}$ is the reduction of $\delta_{p}$ [17, Proposition 2.14]. There is a dual coaction $\widehat{\delta}$ of $\left(\widehat{S}_{V}\right)_{p}$ on the full crossed product $A \times_{\delta} \widehat{S}_{V}$ which satisfies

$$
\widehat{\delta}(j(a) \mu(y))=(j(a) \otimes 1)(\mu \otimes \mathrm{id})\left(\widehat{\delta}_{V}(y)\right)
$$

for all $a \in A, y \in\left(\widehat{S}_{V}\right)_{p}$; we also denote its reduction by $\widehat{\delta}$ [19, Proposition 2.13].

Now suppose $V$ comes from a $\operatorname{Kac}$ system $(H, V, U)$ [2, Définition 6.4]. For any coaction $\delta$ of $S_{V}$ on $A$, we have

$$
A \times_{\delta, r} \widehat{S}_{V}=\overline{\operatorname{span}}\left\{\pi_{L}(a)(1 \otimes \rho(\omega)) \mid a \in A, \omega \in \mathcal{L}(H) * \subseteq \mathcal{L}_{A}(A \otimes H)\right.
$$


Similarly, for any coaction $\delta$ of $\widehat{S}_{V}$ on $A$, we denote the reduced crossed product by $A \times_{\delta, r} S_{V}[2$, Définition 7.1], and we have

$$
A \times_{\delta, r} S_{V}=\overline{\operatorname{span}}\left\{\widehat{\pi}_{\lambda}(a)(1 \otimes L(\omega)) \mid a \in A, \omega \in \mathcal{L}(H)_{*}\right\} \subseteq \mathcal{L}_{A}(A \otimes H),
$$

where $\lambda=\operatorname{Ad}(U) \circ \rho$ and $\widehat{\pi}_{\lambda}=($ id $\otimes \lambda) \circ \delta[2$, Lemme 7.2].

It is important to note that for any $\operatorname{Kac}$ system $(H, V, U),(H, \widehat{V}, U)$ is also a Kac system [2, Proposition 6.5], where $\widehat{V}=\Sigma(U \otimes 1) V(U \otimes 1) \Sigma$ and $\Sigma$ denotes the flip operator on $H \otimes H$, and that then $\left(\widehat{S}_{V}, \widehat{\delta}_{V}\right) \cong\left(S_{\widehat{V}}, \delta_{\widehat{V}}\right)$ as a consequence of [2, Proposition 6.7]. Hence, for any coaction $\left(A, \widehat{S}_{V}, \delta\right)$ there is a coaction $\left(A, S_{\widehat{V}}, \delta^{\prime}\right)$ such that $A \times_{\delta} S_{V} \cong$ $A \times_{\delta^{\prime}} \widehat{S}_{\widehat{V}}$ and $A \times_{\delta, r} S_{V} \cong A \times_{\delta^{\prime}, r} \widehat{S}_{\widehat{V}}$. Thus any results about crossed products by coactions of $S_{V}$ always yield analogous results for coactions of $\widehat{S}_{V}$ : for example, Equation (1.2) above can be derived from Equation (1.1) by replacing $V$ by $\widehat{V}$.

Let $V$ be a regular multiplicative unitary, and let $\psi: A \rightarrow M(B)$ be a nondegenerate homomorphism which is equivariant for coactions $\delta_{A}$ and $\delta_{B}$ of $S_{V}$; that is, such that

$$
\delta_{B} \circ \psi=(\psi \otimes \mathrm{id}) \circ \delta_{A}
$$

If $\left(j_{B}, v_{B}\right)$ is the universal covariant pair for $\left(B, S_{V}, \delta_{B}\right)$ on $B \times \widehat{S}_{V}$, then $\left(j_{B} \circ \psi, v_{B}\right)$ is a covariant pair for $\left(A, S_{V}, \delta_{A}\right)$, so we get a nondegenerate homomorphism $\psi \times \widehat{S}_{V}=$ $\left(j_{B} \circ \psi\right) \times \mu_{B}: A \times \widehat{S}_{V} \rightarrow M\left(B \times \widehat{S}_{V}\right)$, where $\mu_{B}:\left(\widehat{S}_{V}\right)_{p} \rightarrow M\left(A \times \widehat{S}_{V}\right)$ corresponds to $v_{B}$ as in [17, Lemma 2.6]. If $\psi$ is equivariant for coactions of $\widehat{S}_{V}$ on $A$ and $B$, we likewise get a nondegenerate homomorphism $\psi \times S_{V}: A \times S_{V} \rightarrow M\left(B \times S_{V}\right)$.

The analogous result for reduced crossed products, which we shall need in order to define the reduced right-Hilbert bimodule crossed products in the next section, requires a bit more work:

Lemma 1.1. Let $V$ be a regular multiplicative unitary on a Hilbert space $H$, and let $\delta_{A}$ and $\delta_{B}$ be coactions of $S_{V}$ on $C^{*}$-algebras $A$ and $B$. Suppose also that $\psi: A \rightarrow M(B)$ is a $\delta_{A}-\delta_{B}$ equivariant nondegenerate homomorphism. Then there exists a nondegenerate homomorphism $\psi \times_{r} \widehat{S}_{V}: A \times_{\delta_{A}, r} \widehat{S}_{V} \rightarrow M\left(B \times_{\delta_{B}, r} \widehat{S}_{V}\right)$ such that

$$
\left(\psi \times_{r} \widehat{S}_{V}\right)\left(\pi_{L}^{A}(a)(1 \otimes \rho(y))\right)=\pi_{L}^{B}(\psi(a))(1 \otimes \rho(y))
$$

for $a \in A$ and $y \in \widehat{S}_{V}$.

Proof: As in the proof of [2, Théorème 7.5], $A \times_{r} \widehat{S}_{V}$ acts nondegenerately on $A \otimes H$, and therefore on $(A \otimes H) \otimes_{A} B$, where $B$ is the standard right-Hilbert $A-B$ bimodule arising from $\psi$. It is straightforward to check that the map $\Phi:(A \otimes H) \otimes_{A} B \rightarrow B \otimes H$ determined by

$$
\Phi\left((a \otimes \xi) \otimes_{A} b\right)=\psi(a) b \otimes \xi
$$


is a Hilbert $B$-module isomorphism; thus $\mathcal{L}_{B}\left((A \otimes H) \otimes_{A} B\right) \cong \mathcal{L}_{B}(B \otimes H)$, so we obtain a nondegenerate homomorphism $\psi \times_{\mathrm{r}} \widehat{S}_{V}: A \times_{\mathrm{r}} \widehat{S}_{V} \rightarrow \mathcal{L}_{B}(B \otimes H)$ characterised by

$$
\left(\psi \times_{r} \widehat{S}_{V}\right)\left(\pi_{L}^{A}(a)(1 \otimes \rho(y))\right)\left(\Phi\left((c \otimes \xi) \otimes_{A} b\right)\right)=\Phi\left(\pi_{L}^{A}(a)(1 \otimes \rho(y))\left((c \otimes \xi) \otimes_{A} b\right)\right)
$$

for $a, c \in A, y \in \widehat{S}_{V}, \xi \in H$, and $b \in B$.

Now for $a, c, \xi$, and $b$ as above, factor $\xi=L(x) \eta$ for some $x \in S_{V}$ and $\eta \in H$, and choose $a_{i} \in A$ and $x_{i} \in S_{V}$ such that $\delta_{A}(a)(1 \otimes x) \approx \sum_{i}^{n} a_{i} \otimes x_{i}$. Then we have

$$
\begin{aligned}
\left(\psi \times_{r} \widehat{S}_{V}\right)\left(\pi_{L}^{A}(a)\right)\left(\Phi\left((c \otimes \xi) \otimes_{A} b\right)\right) & =\Phi\left(\pi_{L}^{A}(a)(c \otimes \xi) \otimes_{A} b\right) \\
& =\Phi\left((\mathrm{id} \otimes L) \circ \delta_{A}(a)(c \otimes L(x) \eta) \otimes_{A} b\right) \\
& =\Phi\left((\mathrm{id} \otimes L)\left(\delta_{A}(a)(1 \otimes x)\right)(c \otimes \eta) \otimes_{A} b\right) \\
& \approx \sum_{i}^{n} \Phi\left((\mathrm{id} \otimes L)\left(a_{i} \otimes x_{i}\right)(c \otimes \eta) \otimes_{A} b\right) \\
& =\sum_{i}^{n} \Phi\left(\left(a_{i} \otimes L\left(x_{i}\right)\right)(c \otimes \eta) \otimes \otimes_{A} b\right) \\
& =\sum_{i}^{n} \Phi\left(\left(a_{i} c \otimes L\left(x_{i}\right) \eta\right) \otimes{ }_{A} b\right) \\
& =\sum_{i}^{n} \psi\left(a_{i} c\right) b \otimes L\left(x_{i}\right) \eta \\
& =\sum_{i}^{n}\left(\psi\left(a_{i}\right) \otimes L\left(x_{i}\right)\right)(\psi(c) b \otimes \eta) \\
& =(\psi \otimes L)\left(\sum_{i}^{n} a_{i} \otimes x_{i}\right)(\psi(c) b \otimes \eta) \\
& \approx(\psi \otimes L)\left(\delta_{A}(a)(1 \otimes x)\right)(\psi(c) b \otimes \eta) \\
& =(\psi \otimes L)\left(\delta_{A}(a)\right)(1 \otimes L(x))(\psi(c) b \otimes \eta) \\
& =(\psi \otimes L) \circ \delta_{A}(a)(\psi(c) b \otimes \xi) \\
& =(\mathrm{id} \otimes L) \circ \delta_{B}(\psi(a)) \Phi\left((c \otimes \xi) \otimes \otimes_{B} b\right) \\
& =\pi_{L}^{B}(\psi(a))\left(\Phi\left((c \otimes \xi) \otimes \theta_{B} b\right)\right) \\
&
\end{aligned}
$$

so that $\left(\psi \times_{r} \widehat{S}_{V}\right)\left(\pi_{L}^{A}(a)\right)=\pi_{L}^{B}(\psi(a))$. Since it is straightforward to check that $\left(\psi \times_{r}\right.$ $\left.\widehat{S}_{V}\right)(1 \otimes \rho(y))=1 \otimes \rho(y)$ for $y \in \widehat{S}_{V}$, this shows that $\psi \times_{r} \widehat{S}_{V}$ maps $A \times_{r} \widehat{S}_{V}$ into $M\left(B \times{ }_{r} \widehat{S}_{V}\right) \subseteq \mathcal{L}_{B}(B \otimes H)$ and also establishes Equation (1.3), which in turn makes it evident that $\psi \times{ }_{r} \widehat{S}_{V}$ is nondegenerate. 


\section{CoACTIONS ON RIGHT-HILBERT BIMODULES}

Let $V$ be a regular multiplicative unitary. For simplicity, we shall just write $S$ for $S_{V}$ and $\widehat{S}$ for $\widehat{S}_{V}$. We define a coaction of the Hopf $C^{*}$-algebra $S$ on a right-Hilbert $A$ - $B$ bimodule $X$ to be an imprimitivity bimodule coaction $\left(\delta_{K}, \delta_{X}, \delta_{B}\right)$ of $S$ on ${ }_{K} X_{B}$ [16, Definition 3.3(a)] together with a $C^{*}$-coaction $\delta_{A}$ of $S$ on $A$ such that the associated homomorphism $\psi: A \rightarrow M(K)$ is $\delta_{A}-\delta_{K}$ equivariant. We say that a right-Hilbert bimodule coaction $\left(\delta_{A}, \delta_{X}, \delta_{B}\right)$ is injective if $\delta_{A}$ and $\delta_{B}$ are (in which case $\delta_{X}$ will be also), and we say it is nondegenerate if $\delta_{A}$ and $\delta_{B}$ are nondegenerate $C^{*}$-coactions.

Given an imprimitivity bimodule coaction $\left(\delta_{K}, \delta_{X}, \delta_{B}\right)$ of $S$ on ${ }_{K} X_{B}$, the rule

$$
\delta_{L}\left(\begin{array}{ll}
k & x \\
\tilde{y} & b
\end{array}\right)=\left(\begin{array}{ll}
\delta_{K}(k) & \delta_{X}(x) \\
\delta_{X}(y) & \delta_{B}(b)
\end{array}\right)
$$

defines a coaction $\delta_{L}$ of $S$ on the linking algebra $L(X)=\left(\begin{array}{ll}K & X \\ \widetilde{X} & B\end{array}\right)$ [16, Lemma 3.7]. Departing slightly from $\mathrm{Ng}$, we shall define the imprimitivity bimodule crossed product ${ }_{K} X_{B} \times{ }_{\delta_{X}} \widehat{S}$ to be the corner $j_{L}(p)\left(L(X) \times \times_{\delta_{L}} \widehat{S}\right) j_{L}(q)$, where $p=\left(\begin{array}{ll}1 & 0 \\ 0 & 0\end{array}\right)$ and $q=\left(\begin{array}{ll}0 & 0 \\ 0 & 1\end{array}\right)$ are the canonical projections in $M(L(X))$. By [16, Theorem 3.11], ${ }_{K} X_{B} \times_{\delta_{X}} \widehat{S}$ is then a $K \times_{\delta_{K}} \widehat{S}-B \times_{\delta_{B}} \widehat{S}$ imprimitivity bimodule which is an imprimitivity bimodule crossed product in Ng's sense [16, Definition 3.5(b)], and we have

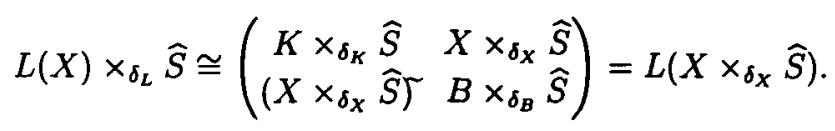

Similarly (this time in keeping with $\mathrm{Ng}$ ), we define the reduced crossed product ${ }_{K} X_{B} \times_{\delta_{X}, r}$ $\widehat{S}$ to be $($ id $\otimes L) \circ \delta_{L}(p)\left(L(X) \times_{\delta_{L}, r} \widehat{S}\right)($ id $\otimes L) \circ \delta_{L}(q)[16$, Remark 3.20(a)]. By the proof of [16, Proposition 3.19], it is a $K \times_{\delta_{K}, r} \widehat{S}-B \times_{\delta_{B}, r} \widehat{S}$ imprimitivity bimodule, and

$$
L(X) \times_{\delta_{L}, r} \widehat{S} \cong\left(\begin{array}{ccc}
K \times_{\delta_{K}, r} \widehat{S} & X \times_{\delta_{X}, r} \widehat{S} \\
\left(X \times_{\delta_{X}, r} \widehat{S}\right) & B \times_{\delta_{B}, r}
\end{array}\right)=L\left(X \times_{\delta_{X}, r} \widehat{S}\right) .
$$

Now given a right-Hilbert bimodule coaction $\left(\delta_{A}, \delta_{X}, \delta_{B}\right)$ of $S$ on ${ }_{A} X_{B}$, the nondegenerate homomorphism $\psi \times \widehat{S}: A \times \widehat{S} \rightarrow M(K \times \widehat{S})$ makes ${ }_{K} X_{B} \times \widehat{S}$ into a rightHilbert $A \times \widehat{S}-B \times \widehat{S}$ bimodule, which we denote by ${ }_{A} X_{B} \times \widehat{S}$ and call the right-Hilbert bimodule crossed product of ${ }_{A} X_{B}$ by $S$. Similarly, the nondegenerate homomorphism $\psi \times_{r} \widehat{S}: A \times_{r} \widehat{S} \rightarrow M\left(K \times_{r} \widehat{S}\right)$ of Lemma 1.1 makes ${ }_{K} X_{B} \times_{r} \widehat{S}$ into a right-Hilbert $A \times_{r} \widehat{S}$ $-B \times_{T} \widehat{S}$ bimodule, which we denote ${ }_{A} X_{B} \times_{T} \widehat{S}$. If $V$ comes from a Kac system, we define right-Hilbert bimodule coactions of $\widehat{S}_{V} \cong S_{\widehat{V}}$ and the right-Hilbert bimodule crossed products ${ }_{A} X_{B} \times S_{V}$ and ${ }_{A} X_{B} \times{ }_{r} S_{V}$ by replacing $V$ with $\widehat{V}$ in the above definitions.

If $\left(\delta_{K}, \delta_{X}, \delta_{B}\right)$ is an imprimitivity bimodule coaction of $S$ on ${ }_{K} X_{B}$, it is straightforward to check that the dual coaction $\widehat{\delta}_{L}$ of $\widehat{S}_{p}$ on $L(X) \times \widehat{S}$ restricts to the dual coactions 
$\widehat{\delta}_{K}$ and $\widehat{\delta}_{B}$ on the diagonal corners $K \times \widehat{S}$ and $B \times \widehat{S}$. The restriction of $\widehat{\delta}_{L}$ to the upper right corner ${ }_{K} X_{B} \times \widehat{S}$ gives a map $\widehat{\delta}_{X}$ such that $\left(\widehat{\delta}_{K}, \widehat{\delta}_{X}, \widehat{\delta}_{B}\right)$ is an imprimitivity bimodule coaction of $\widehat{S}_{p}$ on ${ }_{K} X_{B} \times \widehat{S}$ which we call the dual imprimitivity bimodule coaction. (One can show that this definition agrees with that given for Hilbert modules in [16, Remark 2.18].) If $\left(\delta_{A}, \delta_{X}, \delta_{B}\right)$ is a right-Hilbert bimodule coaction of $S$ on ${ }_{A} X_{B}$, we define the dual coaction of $\widehat{S}_{p}$ on ${ }_{A} X_{B} \times \widehat{S}$ to be the dual imprimitivity bimodule coaction $\left(\widehat{\delta}_{K}, \widehat{\delta}_{X}, \widehat{\delta}_{B}\right)$, together with the dual $C^{*}$-coaction $\widehat{\delta}_{A}$. Since

$$
\begin{aligned}
((\psi \times \widehat{S}) \otimes \mathrm{id}) \circ \widehat{\delta}_{A}\left(j_{A}(a) \mu_{A}(y)\right) & =((\psi \times \widehat{S}) \otimes \mathrm{id})\left(\left(j_{A}(a) \otimes 1\right)\left(\mu_{A} \otimes \mathrm{id}\right)\left(\widehat{\delta}_{V}(y)\right)\right) \\
& =\left(j_{K}(\psi(a)) \otimes 1\right)\left(\mu_{K} \otimes \mathrm{id}\right)\left(\widehat{\delta}_{V}(y)\right) \\
& =\widehat{\delta}_{K}\left(j_{K}(\psi(a)) \mu_{K}(y)\right) \\
& =\widehat{\delta}_{K} \circ(\psi \times \widehat{S})\left(j_{A}(a) \mu_{A}(y)\right)
\end{aligned}
$$

for all $a \in A, y \in \widehat{S}_{p}$, the nondegenerate homomorphism $\psi \times \widehat{S}: A \times \widehat{S} \rightarrow M(K \times \widehat{S})$ is $\widehat{\delta}_{A}-\widehat{\delta}_{K}$ equivariant, so this is indeed a right-Hilbert bimodule coaction.

Given a right-Hilbert bimodule coaction $\left(\delta_{A}, \delta_{K}, \delta_{K}\right)$ of $S$ on a standard bimodule ${ }_{A} K_{K}$, we have potentially two different right-Hilbert $A \times \widehat{S}-K \times \widehat{S}$ bimodules: the bimodule crossed product ${ }_{A} K_{K} \times \widehat{S}$ and the standard bimodule formed from the $C^{*}$ algebra crossed product $K \times \widehat{S}$ and the nondegenerate homomorphism $\psi \times \widehat{S}: A \times \widehat{S} \rightarrow$ $M(K \times \widehat{S})$. The following lemma shows that these coincide; in other words, a crossed product of a standard bimodule is a standard bimodule.

LEMMA 2.1. Let $V$ be a regular multiplicative unitary, and let $\left(\delta_{A}, \delta_{K}, \delta_{K}\right)$ be a right-Hilbert bimodule coaction of $S=S_{V}$ on a standard bimodule ${ }_{A} K_{K}$. Then the rightHilbert bimodule crossed product ${ }_{A} K_{K} \times{ }_{\delta_{K}} \widehat{S}$ is isomorphic to the $C^{*}$-crossed product $K \times_{\delta_{K}} \widehat{S}$ as a right-Hilbert $A \times_{\delta_{A}} \widehat{S}-K \times_{\delta_{K}} \widehat{S}$ bimodule. An analogous statement also holds for the reduced crossed products.

Proof: Since ${ }_{A} K_{K} \times \widehat{S}$ is by definition ${ }_{K} K_{K} \times \widehat{S}$ with the same homomorphism $\psi \times \widehat{S}$, it suffices to show that ${ }_{K} K_{K} \times \widehat{S}$ is isomorphic to the $C^{*}$-crossed product $K \times_{\delta_{K}} \widehat{S}$ as a $K \times_{\delta_{K}} \widehat{S}-K \times_{\delta_{K}} \widehat{S}$ imprimitivity bimodule. Let $L=\left(\begin{array}{ll}K & K \\ K & K\end{array}\right)$ be the linking algebra for $K_{K} K_{K}$, and let $\delta_{L}=\left(\begin{array}{ll}\delta_{K} & \delta_{K} \\ \delta_{K} & \delta_{K}\end{array}\right)$ be the associated coaction. Then by [16, Theorem 3.11],

$$
L \times_{\delta_{L}} \widehat{S} \cong\left(\begin{array}{cc}
K \times \widehat{S} & K K_{K} \times \widehat{S} \\
K K_{K} \times \widehat{S} & K \times \widehat{S}
\end{array}\right)
$$

and the projection $j\left(\begin{array}{cc}1_{K} & 0 \\ 0 & 0\end{array}\right)$ in $M\left(L \times \times_{\delta_{L}} \hat{S}\right)$ corresponds to $\left(\begin{array}{cc}1_{K \times \widehat{S}} & 0 \\ 0 & 0\end{array}\right)$ under this
isomorphism. 
Let $M_{2}$ denote the $C^{*}$-algebra of two-by-two matrices over $\mathbb{C}$. Then the canonical isomorphism $\Phi: M_{2} \otimes K \rightarrow L$ determined by $\Phi\left(\left(\begin{array}{ll}a & b \\ c & d\end{array}\right) \otimes k\right)=\left(\begin{array}{ll}a k & b k \\ c k & d k\end{array}\right)$ is clearly
id $\otimes \delta_{K}-\delta_{K}$ equivariant; thus

$$
L \times_{\delta_{L}} \widehat{S} \cong\left(M_{2} \otimes K\right) \times_{\mathrm{id} \otimes \delta_{K}} \widehat{S} .
$$

Note that this isomorphism takes $j\left(\begin{array}{cc}1_{K} & 0 \\ 0 & 0\end{array}\right) \in M\left(L \times_{\delta_{L}} \widehat{S}\right)$ to $j\left(\left(\begin{array}{ll}1 & 0 \\ 0 & 0\end{array}\right) \otimes 1_{K}\right) \epsilon$
$M\left(\left(M_{2} \otimes K\right) \times_{\mathrm{id} \otimes \delta_{K}} \widehat{S}\right)$. We next claim that

$$
\left(M_{2} \otimes K\right) \times_{\mathrm{id} \otimes \delta_{K}} \widehat{S} \cong M_{2} \otimes\left(K \times_{\delta_{K}} \widehat{S}\right)
$$

For if $\iota$ denotes the trivial coaction of $\mathbb{C}$ on $M_{2}$, then [17, Proposition 3.2] implies that

$$
\left(M_{2} \otimes K\right) \times_{\iota \otimes \delta_{K}} \widehat{\mathbb{C} \otimes S} \cong\left(M_{2} \times_{\iota} \widehat{\mathbb{C}}\right) \otimes\left(K \times_{\delta_{K}} \widehat{S}\right)
$$

and the right and left sides of this equation are naturally isomorphic to the right and left sides, respectively, of Equation (2.3). Under this isomorphism, the projection $j\left(\left(\begin{array}{ll}1 & 0 \\ 0 & 0\end{array}\right) \otimes 1_{K}\right) \in M\left(\left(M_{2} \otimes K\right) \times_{\mathrm{id} \otimes \delta_{K}} \widehat{S}\right)$ is carried to $\left(\begin{array}{ll}1 & 0 \\ 0 & 0\end{array}\right) \otimes 1_{K \times \widehat{S}} \in$ $M\left(M_{2} \otimes\left(K \times_{\delta_{K}} \widehat{S}\right)\right)$.

Combining Equations (2.1), (2.2), and (2.3), we have

$$
\left(\begin{array}{cc}
K \times \widehat{S} & K_{K} \times \widehat{S} \\
K K_{K} \times \widehat{S} & K \times \widehat{S}
\end{array}\right) \cong M_{2} \otimes\left(K \times_{\delta_{K}} \widehat{S}\right)
$$

and since $j\left(\begin{array}{cc}1_{K} & 0 \\ 0 & 0\end{array}\right)$ maps to $\left(\begin{array}{ll}1 & 0 \\ 0 & 0\end{array}\right) \otimes 1$, it follows that the corners $K K_{K} \times_{\delta_{K}} \widehat{S}$ and $K \times_{\delta_{K}} \widehat{S}$ are isomorphic as $K \times_{\delta_{K}} \widehat{S}-K \times_{\delta_{K}} \widehat{S}$ imprimitivity bimodules.

For the reduced crossed products, it again suffices to show that ${ }_{K} K_{K} \times{ }_{r} \widehat{S}$ is isomorphic to $K \times_{r} \widehat{S}$ as a $K \times_{r} \widehat{S}-K \times_{r} \widehat{S}$ imprimitivity bimodule. By [16, Remark 3.20(a)] we have

$$
L \times_{\delta_{L}, r} \widehat{S} \cong\left(\begin{array}{cc}
K \times{ }_{r} \widehat{S} & { }_{K} K_{K} \times{ }_{r} \widehat{S} \\
K K_{K} \times{ }_{r} \widehat{S} & K \times{ }_{r} \widehat{S}
\end{array}\right),
$$

and it follows from equivariance of $\Phi: M_{2} \otimes K \rightarrow L$ that

$$
L \times_{\delta_{L}, r} \widehat{S} \cong\left(M_{2} \otimes K\right) \times_{\mathrm{id} \otimes \delta_{K}, r} \widehat{S}
$$

Applying [17, Proposition 3.3], which is the reduced version of [17, Proposition 3.2], we get

$$
\left(M_{2} \otimes K\right) \times_{\iota \otimes \delta_{K}, r} \widehat{\mathbf{C} \otimes S} \cong\left(M_{2} \times_{\iota, r} \widehat{\mathbb{C}}\right) \otimes\left(K \times_{\delta_{K}, r} \widehat{S}\right)
$$


and hence

$$
\left(M_{2} \otimes K\right) \times{ }_{r} \widehat{S} \cong M_{2} \otimes(K \times, \widehat{S}) .
$$

Combining these isomorphisms and matching up the projections as above, it follows that ${ }_{K} K_{K} \times_{\delta_{K}, r} \widehat{S} \cong K \times_{\delta_{K}, r} \widehat{S}$.

For the proof of our main result (Theorem 4.1) we shall need to know that the decomposition ${ }_{A} X_{B} \cong{ }_{A} K \otimes_{K} X_{B}$ is equivariant:

LEMMA 2.2. Let $V$ be a regular multiplicative unitary, let $\left(\delta_{A}, \delta_{X}, \delta_{B}\right)$ be a rightHilbert bimodule coaction of $S=S_{V}$ on ${ }_{A} X_{B}$, and let $\delta_{K}$ be the associated coaction on $K=\mathcal{K}_{B}(X)$. Then there exist right-Hilbert bimodule isomorphisms

$$
{ }_{A} X_{B} \times{ }_{\delta_{X}} \widehat{S} \cong\left({ }_{A} K_{K} \times{ }_{\delta_{K}} \widehat{S}\right) \otimes_{K \times \widehat{S}}\left(K_{K} X_{B} \times{ }_{\delta_{X}} \widehat{S}\right)
$$

and

$$
{ }_{A} X_{B} \times \delta_{\delta_{X}, r} \widehat{S} \cong\left({ }_{A} K_{K} \times \times_{\delta_{K}, r} \widehat{S}\right) \otimes_{K \times_{r} \hat{S}}\left({ }_{K} X_{B} \times{ }_{\delta_{X}, r} \widehat{S}\right) .
$$

Proof: By definition, ${ }_{A} X_{B} \times{ }_{\delta_{X}} \widehat{S}$ is the imprimitivity bimodule ${ }_{K} X_{B} \times{ }_{\delta_{X}} \widehat{S}$ with the nondegenerate homomorphism $\psi \times \widehat{S}: A \times \widehat{S} \rightarrow M(K \times \widehat{S})$ arising from $\psi: A \rightarrow M(K)$. Since ${ }_{A} K_{K} \times \widehat{S}$ is ${ }_{K} K_{K} \times \widehat{S}$ with the same map, for the first isomorphism it suffices to show that ${ }_{K} X_{B} \times_{\delta_{X}} \widehat{S} \cong\left({ }_{K} K_{K} \times_{\delta_{K}} \widehat{S}\right) \otimes_{K \times \hat{S}}\left({ }_{K} X_{B} \times_{\delta_{X}} \widehat{S}\right)$ as imprimitivity bimodules. But by Lemma $2.1,{ }_{K} K_{K} \times \widehat{S} \cong K \times \widehat{S}$, so the result follows from the usual cancellation $C \otimes_{C} Y \cong Y$.

The assertion about the reduced crossed products follows similarly from Lemma 2.1.

\section{THE FIXED-POINT THEOREM}

Based upon the familiar results for actions of compact groups and coactions of discrete groups, one would guess that the crossed product by a coaction of a Hopf $C^{*}$-algebra of compact type is Morita equivalent to the fixed-point algebra. In [18] $\mathrm{Ng}$ proves a version of this fixed-point theorem, and this is crucial for his imprimitivity theorem, which we study in the next section. (We should point out that the imprimitivity theorem naturally involves a coaction of $\widehat{S}_{U}$ for a multiplicative unitary $U$ coming from a discrete Kac system, but is proved by applying the fixed-point theorem to the corresponding coaction of $S_{\widehat{U}}$, where $\widehat{U}$ is compact.) Here we recall Ng's fixed-point result and establish some relations to multipliers and bimodules, in preparation for our work with $\mathrm{Ng}$ 's imprimitivity theorem in Section 4.

Let $V$ be a regular multiplicative unitary of compact type such that $S_{V}$ has a faithful Haar state $\varphi$. Again we shall just write $S$ for $S_{V}$ and $\widehat{S}$ for $\widehat{S}_{V}$. Let $\delta$ be a coaction of $S$ on a $C^{*}$-algebra $A$ which is effective in the sense that

$$
\overline{\operatorname{span}}\{\delta(A)(A \otimes 1)\}=A \otimes S .
$$


$\mathrm{Ng}$ shows in two steps [18, Theorem 2.7 and Proposition 2.9] that the reduced crossed product $A \times_{\delta, r} \widehat{S}$ is Morita equivalent to the fixed-point algebra $A^{\delta}$. Since it will simplify our computations with the imprimitivity bimodule, we shall combine Ng's two steps into one.

$\mathrm{Ng}$ 's strategy is to use a nonunital version of Watatani's $C^{*}$-basic construction [22]. The map $E=E_{A}=(\mathrm{id} \otimes \varphi) \circ \delta$ is a conditional expectation of $A$ onto $A^{\delta}$, and so $A$ becomes a full pre-Hilbert $A^{\delta}$-module under right multiplication and the pre-inner product

$$
\langle a, b\rangle_{A^{\delta}}=E\left(a^{*} b\right) \text {. }
$$

The Hausdorff completion of the pre-Hilbert $A^{\delta}$-module $A$ is a full Hilbert $A^{\delta}$-module, denoted $\mathcal{F}=\mathcal{F}(A)$. Let $\eta=\eta_{A}$ be the canonical map of $A$ into $\mathcal{F}$, and define $e_{A} \in \mathcal{L}_{A^{\delta}}(\mathcal{F})$ and $\lambda=\lambda_{A}: A \rightarrow \mathcal{L}_{A^{\delta}}(\mathcal{F})$ by

$$
e_{A} \eta(a)=\eta(E(a)) \text { and } \lambda(a) \eta(b)=\eta(a b) .
$$

Then the $C^{*}$-basic construction is defined to be the closed span in $\mathcal{L}_{A^{\delta}}(\mathcal{F})$ of $\lambda(A) e_{A} \lambda(A)$, and is denoted $C^{*}\left\langle A, e_{A}\right\rangle$. Since

$$
e_{A} \lambda(a) e_{A}=\lambda(E(a)) e_{A} \text { and } E\left(a^{*}\right)=E(a)^{*},
$$

$C^{*}\left\langle A, e_{A}\right\rangle$ is a $C^{*}$-algebra; in fact, a routine computation shows $C^{*}\left\langle A, e_{A}\right\rangle$ coincides with the imprimitivity algebra $\mathcal{K}_{A^{\delta}}(\mathcal{F})$. Moreover, a short computation shows that the left inner product is given on the generators by

$$
C^{*}\left\langle A, e_{A}\right\rangle\langle\eta(a), \eta(b)\rangle=\lambda(a) e_{A} \lambda\left(b^{*}\right) .
$$

Therefore, the Hausdorff completion $\mathcal{F}$ of the $\operatorname{span}\left\{\lambda(A) e_{A} \lambda(A)\right\}-A^{\delta}$ pre-imprimitivity bimodule $A$ is a $C^{*}\left\langle A, e_{A}\right\rangle-A^{\delta}$ imprimitivity bimodule.

$\mathrm{Ng}$ 's first step is to temporarily assume the coaction $\delta$ is injective. Then the conditional expectation $E$ is faithful, and $\mathrm{Ng}$ proves [18, Theorem 2.7] that in this case the map

$$
\lambda(a) e_{A} \lambda(b) \mapsto \delta(a)(1 \otimes \rho(\varphi)) \delta(b)
$$

extends to an isomorphism of the $C^{*}$-basic construction $C^{*}\left\langle A, e_{A}\right\rangle$ onto the reduced crossed product $A \times_{\delta, r} \widehat{S}$, where

$$
\rho(\varphi)=(\operatorname{id} \otimes \varphi)(V),
$$

which, as Ng observes in [18, proof of Lemma 2.5], is a member of $\widehat{S}$.

$\mathrm{Ng}$ 's second step is to remove the injectivity condition on $\delta$ and note that, if we put $I=\operatorname{ker} \delta$, there is an injective coaction $\delta^{\prime}$ on $A / I$ given by $\delta^{\prime}(q(a))=(q \otimes \mathrm{id}) \circ \delta(a)$, where 
$q: A \rightarrow A / I$ is the quotient map. Then $\delta^{\prime}$ is also effective, $\dot{q}$ maps $A^{\delta}$ isomorphically onto $(A / I)^{\delta^{\prime}}$, and the reduced crossed products $A \times_{\delta, r} \widehat{S}$ and $(A / I) \times_{\delta^{\prime}, r} \widehat{S}$ coincide. $\mathrm{Ng}$ deduces as a corollary [18, Proposition 2.9] that $A \times_{\delta, r} \widehat{S}$ is still Morita equivalent to $A^{\delta}$.

To combine Ng's two steps, note that in the second step the imprimitivity bimodule $\mathcal{F}(A / I)$ is the completion of $A / I$ with inner product

$$
\langle q(a), q(b)\rangle_{(A / I)^{\delta^{\prime}}}=E_{A / I}\left(q(a)^{*} q(b)\right)=q \circ E_{A}\left(a^{*} b\right)=q\left(\langle a, b\rangle_{A^{\delta}}\right) .
$$

Since $q$ is faithful on the image of $\langle\cdot, \cdot\rangle_{A^{\delta}}, \mathcal{F}(A / I)$ can be identified with $\mathcal{F}(A)$. More precisely, the map $\eta_{A}(a) \mapsto \eta_{A / I}(q(a))$ is well-defined and extends to an isomorphism $\Phi$ of the Hilbert $A^{\delta}$-module $\mathcal{F}(A)$ onto the Hilbert $(A / I)^{\delta^{\prime}}$-module $\mathcal{F}(A / I)$, with right coefficient map $\left.q\right|_{A^{\delta}}$. Moreover, a short computation shows

$$
\Phi\left(\lambda_{A}(a) e_{A} \lambda_{A}(b) \eta_{A}(c)\right)=\lambda_{A / I}(q(a)) e_{A / I} \lambda_{A / I}(q(b)) \Phi\left(\eta_{A}(c)\right),
$$

so $\Phi$ is in fact an isomorphism of the $C^{*}\left\langle A, e_{A}\right\rangle-A^{\delta}$ imprimitivity bimodule $\mathcal{F}(A)$ onto the $C^{*}\left\langle A / I, e_{A / I}\right\rangle-(A / I)^{\delta^{\prime}}$ imprimitivity bimodule $\mathcal{F}(A / I)$, with left coefficient map determined by

$$
\lambda_{A}(a) e_{A} \lambda_{A}(b) \mapsto \lambda_{A / I}(q(a)) e_{A / I} \lambda_{A / I}(q(b)) .
$$

Combining with the isomorphism

$$
\lambda_{A / I}(q(a)) e_{A / I} \lambda_{A / I}(q(b)) \mapsto \delta^{\prime}(q(a))(1 \otimes \rho(\varphi)) \delta^{\prime}(q(b))
$$

of $C^{*}\left\langle A / I, e_{A / I}\right\rangle$ onto $(A / I) \times_{\delta^{\prime}, r} \widehat{S}$, and with the identification of $A \times_{\delta, r} \widehat{S}$ and $(A / I) \times_{\delta^{\prime}, r}$ $\widehat{S}$, we get an isomorphism

$$
\lambda_{A}(a) e_{A} \lambda(b) \mapsto \delta(a)(1 \otimes \rho(\varphi)) \delta(b)
$$

of $C^{*}\left\langle A, e_{A}\right\rangle$ onto $A \times_{\delta, r} \widehat{S}$. Putting all this together, we have a one-step version of Ng's fixed-point theorem [18, Proposition 2.11] - although we haven't addressed the case of coactions by $S_{p}$ :

PROPOSITION 3.1. [18] If $V$ is a regular multiplicative unitary of compact type such that $S=S_{V}$ has a faithful Haar state $\varphi$, and if $\delta$ is an effective coaction of $S$ on $A$, then $A$ is a pre-imprimitivity bimodule between the pre- $C^{*}$-algebra $B=$ $\operatorname{span}\{\delta(A)(1 \otimes \rho(\varphi)) \delta(A)\}$ and the fixed-point algebra $A^{\delta}$, with operations given for $a, b, c \in A$ and $d \in A^{\delta}$ by

$$
\begin{aligned}
(\delta(a)(1 \otimes \rho(\varphi)) \delta(b)) \cdot c & =a E(b c) \\
a \cdot d & =a d \\
B\langle a, b\rangle & =\delta(a)(1 \otimes \rho(\varphi)) \delta\left(b^{*}\right) \\
\langle a, b\rangle_{A^{\delta}} & =E\left(a^{*} b\right) .
\end{aligned}
$$

Consequently, the Hausdorff completion $\mathcal{F}(A)$ of $A$ is an $A \times_{\delta, r} \widehat{S}-A^{\delta}$ imprimitivity bimodule. 
Now let $\left(\delta_{A}, \delta_{X}, \delta_{B}\right)$ be a coaction of $S$ on an $A-B$ imprimitivity bimodule $X$, let $L=L(X)$ be the linking algebra, and let $\delta_{L}$ be the associated coaction of $S$ on $L$. Then we have

$$
\begin{aligned}
\delta_{L}(L)(L \otimes 1) & \supseteq\left(\begin{array}{cc}
\delta_{A}(A) & \delta_{X}(X) \\
\delta_{X}(X)^{\sim} & \delta_{B}(B)
\end{array}\right)\left(\begin{array}{cc}
A \otimes 1 & 0 \\
0 & B \otimes 1
\end{array}\right) \\
& =\left(\begin{array}{cc}
\delta_{A}(A)(A \otimes 1) & \delta_{X}(X) \cdot(B \otimes 1) \\
\delta_{X}(X)^{-} \cdot(A \otimes 1) & \delta_{B}(B)(B \otimes 1)
\end{array}\right)
\end{aligned}
$$

and

$$
\begin{aligned}
\delta_{X}(X) \cdot(B \otimes 1) & =\delta_{X}(X \cdot B) \cdot(B \otimes 1) \\
& =\delta_{X}(X) \cdot \delta_{B}(B)(B \otimes 1)
\end{aligned}
$$

it follows from this (and by symmetry) that $\delta_{L}$ is effective whenever $\delta_{A}$ and $\delta_{B}$ are.

Let $p=\left(\begin{array}{ll}1 & 0 \\ 0 & 0\end{array}\right) \in M(L)$. We shall need the following result in the next section.

LEMMA 3.2. Let $V$ be a regular multiplicative unitary of compact type such that $S=S_{V}$ has a faithful Haar state $\varphi$, and with notation as above, suppose that $\delta_{A}$ and $\delta_{B}$ are effective. Then the inclusion $A \hookrightarrow L$ extends to an isomorphism $\Phi$ of the $A \times_{\delta_{A}, r} \widehat{S}$ $A^{\delta_{A}}$ imprimitivity bimodule $\mathcal{F}(A)$ onto the $\delta_{L}(p)\left(L \times_{\delta_{L}, r} \widehat{S}\right) \delta_{L}(p)-p L^{\delta_{L}} p$ imprimitivity bimodule $\delta_{L}(p) \cdot \mathcal{F}(L) \cdot p$.

PROOF: Let us first make sure we understand all the components of the statement of the lemma. On the right side of $\mathcal{F}(L)$ we regard $p$ as an element of $M\left(L^{\delta_{L}}\right)$, which naturally embeds in $M(L)$. Since the projections $p$ in $M\left(L^{\delta_{L}}\right)$ and $\delta_{L}(p)=p \otimes 1$ in $M\left(L \times_{r} \widehat{S}\right)$ are full, $\delta_{L}(p) \cdot \mathcal{F}(L) \cdot p$ is indeed a $\delta_{L}(p)\left(L \times_{r} \widehat{S}\right) \delta_{L}(p)-p L^{\delta_{L}} p$ imprimitivity bimodule. We have

$$
\begin{aligned}
\delta_{L}(p)\left(L \times_{r} \widehat{S}\right) \delta_{L}(p) & =(p \otimes 1) \overline{\operatorname{span}}\left\{\delta_{L}(L)\left(1_{M(L)} \otimes \widehat{S}\right)\right\}(p \otimes 1) \\
& =\overline{\operatorname{span}}\left\{(p \otimes 1) \delta_{L}(L)(p \otimes 1)\left(1_{M(A)} \otimes \widehat{S}\right)\right\} \\
& =\overline{\operatorname{span}}\left\{\delta_{L}(p) \delta_{L}(L) \delta_{L}(p)\left(1_{M(A)} \otimes \widehat{S}\right)\right\} \\
& =\overline{\operatorname{span}}\left\{\delta_{L}(p L p)\left(1_{M(A)} \otimes \widehat{S}\right)\right\} \\
& =\overline{\operatorname{span}}\left\{\delta_{A}(A)\left(1_{M(A)} \otimes \widehat{S}\right)\right\} \\
& =A \times_{r} \widehat{S}
\end{aligned}
$$

where we have used $p=1_{M(A)}$. On the other side, since $\left.E_{L}\right|_{A}=E_{A}$ and the natural extension of $E_{L}$ to $M(L)$ is a conditional expectation onto $M\left(L^{\delta_{L}}\right)$, we have

$$
p L^{\delta_{L}} p=p E_{L}(L) p=E_{L}(p L p)=E_{A}(A)=A^{\delta_{A}} .
$$


Thus, it suffices to show the inclusion $A \hookrightarrow L$ respects the right inner products and the left module multiplications. For the inner products, if $a, b \in A$ then

$$
\langle a, b\rangle_{L^{\delta_{L}}}=E_{L}\left(a^{*} b\right)=E_{A}\left(a^{*} b\right)=\langle a, b\rangle_{A^{\delta_{A}}} \text {. }
$$

Turning to the left module multiplications, first note that

$$
\delta_{L}(p)\left(1_{M(L)} \otimes \rho(\varphi)\right) \delta_{L}(p)=p \otimes \rho(\varphi)=1_{M(A)} \otimes \rho(\varphi),
$$

so for $a, b \in A$ we have

$$
\delta_{L}(a)\left(1_{M(L)} \otimes \rho(\varphi)\right) \delta_{L}(b)=\delta_{A}(a)\left(1_{M(A)} \otimes \rho(\varphi)\right) \delta_{A}(b) .
$$

Hence, for $a, b, c \in A$ we have

$$
\begin{aligned}
\left(\delta_{L}(a)\left(1_{M(L)} \otimes \rho(\varphi)\right) \delta_{L}(b)\right) \cdot c & =a E_{L}(b c)=a E_{A}(b c) \\
& =\left(\delta_{A}(a)\left(1_{M(A)} \otimes \rho(\varphi)\right) \delta_{A}(b)\right) \cdot c
\end{aligned}
$$

and we're done.

We'll also need the following lemma concerning standard bimodules.

LEMMA 3.3. Let $V$ be a regular multiplicative unitary of compact type such that $S=S_{V}$ has a faithful Haar state $\varphi$. If $\psi: A \rightarrow M(B)$ is a nondegenerate homomorphism which is equivariant for effective coactions $\delta_{A}$ and $\delta_{B}$ of $S$, then $\psi$ extends to a nondegenerate imprimitivity bimodule homomorphism $\Psi: \mathcal{F}(A) \rightarrow M(\mathcal{F}(B))$ with coefficient maps $\psi \times_{r} \widehat{S}$ and $\left.\psi\right|_{A^{\delta_{A}}}$.

PROOF: By [12, Lemma 5.1], it's enough to show $\psi$ preserves both module multiplications and inner products. For $a, b, c \in A$ and $d \in A^{\delta_{A}}$ we have

$$
\begin{aligned}
\psi\left(\left(\delta_{A}(a)(1 \otimes \rho(\varphi)) \delta_{A}(b)\right) \cdot c\right) & =\psi\left(a E_{A}(b c)\right)=\psi(a) \psi \circ E_{A}(b c) \\
& =\psi(a) E_{B} \circ \psi(b c)=\psi(a) E_{B}(\psi(b) \psi(c)) \\
& =\left(\delta_{B}(\psi(a))(1 \otimes \rho(\varphi)) \delta_{B}(\psi(b))\right) \cdot \psi(c) \\
& =\left(\psi \times_{r} \widehat{S}\right)\left(\delta_{A}(a)(1 \otimes \rho(\varphi)) \delta_{A}(b)\right) \cdot \psi(c) \\
\psi(a \cdot d)=\psi(a d) & =\psi(a) \psi(d)=\psi(a) \cdot \psi(d) \\
M\left(B \times_{r} \widehat{S}\right) & \langle\psi(a), \psi(b)\rangle= \\
& =\left(\psi \times_{B}(\psi(a))(1 \otimes \rho(\varphi)) \delta_{B}(\psi(b))\right. \\
& =\left(\psi \times_{r} \widehat{S}\right)\left(\delta_{A}(a)(1 \otimes \rho(\varphi)) \delta_{A}(b)\right)
\end{aligned}
$$

and

$$
\begin{aligned}
\langle\psi(a), \psi(b)\rangle_{M\left(B^{\delta_{B}}\right)} & =E_{B}\left(\psi(a)^{*} \psi(b)\right)=E_{B} \circ \psi\left(a^{*} b\right) \\
& =\psi \circ E_{A}\left(a^{*} b\right)=\left.\psi\right|_{A^{\delta_{A}}}\left(\langle a, b\rangle_{A^{\delta_{A}}}\right)
\end{aligned}
$$




\section{EQUIVARIANCE AND IMPRIMITIVITY}

We now turn to the result we call Ng's imprimitivity theorem [18, Theorem 3.4]. This is an analogue, for multiplicative unitaries of discrete type (in fact, coming from discrete Kac systems), of Green's and Mansfield's imprimitivity theorems for group actions and coactions, respectively. Our main theorem (Theorem 4.1) says that Ng's theorem is compatible with equivariant right-Hilbert bimodules; we begin by introducing the notation and construction of Ng's imprimitivity bimodule.

Let $U, V$, and $W$ be multiplicative unitaries coming from discrete Kac systems, and assume $W$ is a normal submultiplicative unitary of $V$ and $U$ is the corresponding quotient (see [18, Definition 3.2]); this implies that there exist surjective Hopf *-homomorphisms $L_{V, W}: S_{V} \rightarrow S_{W}$ and $\rho_{V, U}:\left(\widehat{S}_{V}\right)_{p} \rightarrow\left(\widehat{S}_{U}\right)_{p}$. Thus any coaction $\delta$ of $S_{V}$ on $A$ can be restricted to a coaction $\delta \mid=\left(\right.$ id $\left.\otimes L_{V, W}\right) \circ \delta$ of $S_{W}$ on $A$, and any dual coaction $\widehat{\delta}$ of $\left(\widehat{S}_{V}\right)_{p}$ on $A \times_{\delta} \widehat{S}_{V}$ can be restricted to a coaction $\widehat{\delta} \mid=\left(\right.$ id $\left.\otimes \rho_{V, U}\right) \circ \widehat{\delta}$ of $\left(\widehat{S}_{U}\right)_{p}$ on $A \times_{\delta} \widehat{S}_{V}$. We can pass to the corresponding coaction of the reduced $C^{*}$-algebra $\widehat{S}_{U}$ without changing either the crossed product or the fixed-point algebra, and we continue to denote this coaction by $\widehat{\delta}$.

Now assume further that $W$ is amenable, and let $\rho_{W, V}:\left(\widehat{S}_{W}\right)_{p} \rightarrow\left(\widehat{S}_{V}\right)_{p}$ be the Hopf *-homomorphism vouchsafed by the normality of $W$ in $V$. $\mathrm{Ng}$ shows that if $\delta$ is nondegenerate, the nondegenerate homomorphism

$$
\phi_{A}=j_{A}^{V} \times\left(\mu_{A}^{V} \circ \rho_{W, V}\right): A \times_{\delta \mid} \widehat{S}_{W} \rightarrow M\left(A \times_{\delta} \widehat{S}_{V}\right)
$$

is actually an isomorphism of $A \times_{\delta \mid} \widehat{S}_{W}$ onto the fixed-point algebra $\left(A \times_{\delta} \widehat{S}_{V}\right)^{\widehat{\delta}}$, and that the restricted dual coaction $\widehat{\delta} \mid$ of $\widehat{S}_{U}$ is effective. Viewing this as an effective coaction of $S_{\hat{U}}$ with $\hat{U}$ compact, Proposition 3.1 provides an $A \times_{\delta} \widehat{S}_{V} \times_{\delta, r} S_{U}-\left(A \times_{\delta} \widehat{S}_{V}\right)^{\widehat{\delta} \mid}$ imprimitivity bimodule $\mathcal{F}\left(A \times_{\delta} \widehat{S}_{V}\right)$; using the isomorphism $\phi_{A}$, this becomes an $A \times_{\delta} \widehat{S}_{V} \times{ }_{\widehat{\delta} \mid, \Gamma} S_{U}-$ $A \times{ }_{\delta \mid} \widehat{S}_{W}$ imprimitivity bimodule which we denote by $N(A)$.

With notation as below, we define the map $\delta_{X} \mid: X \rightarrow M\left(X \otimes S_{W}\right)$ to be (id $\left.\otimes L_{V, W}\right) \circ$ $\delta_{X}$; it is straightforward to check that then $\left(\delta_{K}\left|, \delta_{X}\right|, \delta_{B} \mid\right)$ is an imprimitivity bimodule coaction of $S_{W}$ on ${ }_{K} X_{B}$ and that $\psi: A \rightarrow M(K)$ is $\delta_{A}\left|-\delta_{K}\right|$ equivariant. We call the resulting right-Hilbert bimodule coaction $\left(\delta_{A}\left|, \delta_{X}\right|, \delta_{B} \mid\right)$ of $S_{W}$ the restricted coaction from $S_{V}$. The restricted dual right-Hilbert bimodule coaction $\left(\widehat{\delta}_{A}\left|, \widehat{\delta}_{X}\right|, \widehat{\delta}_{B} \mid\right)$ of $\left(\widehat{S}_{U}\right)_{p}$ is defined similarly; its reduction to $\widehat{S}_{U}$ is also denoted $\left(\widehat{\delta}_{A}\left|, \widehat{\delta}_{X}\right|, \widehat{\delta}_{B} \mid\right)$.

THEOREM 4.1. Let $U, V$, and $W$ be multiplicative unitaries coming from discrete Kac systems, with $W$ an amenable normal submultiplicative unitary of $V$ and $U$ the corresponding quotient. Let $\left(\delta_{A}, \delta_{X}, \delta_{B}\right)$ be an injective, nondegenerate coaction of $S_{V}$ on a right-Hilbert $A-B$ bimodule $X$, and suppose that the associated coaction $\delta_{K}$ 
on the imprimitivity algebra of $X$ is also nondegenerate. Then the diagram

$$
\begin{aligned}
& A \times_{\delta_{A}} \widehat{S}_{V} \times_{\delta_{A} \mid, r} S_{U} \stackrel{N(A)}{\longrightarrow} A \times_{\delta_{A} \mid} \widehat{S}_{W}\left.{ }_{A} X_{B} \times_{\delta_{X}} \widehat{S}_{V} \times_{\delta_{X} 1, r} S_{U}\right\rfloor \\
& B \times \times_{\delta_{B}} \widehat{S}_{V} \times_{\bar{\delta}_{B} \mid, r} S_{U} \underset{N(B)}{\longrightarrow} B \times_{\delta_{B} \mid} \widehat{S}_{W}
\end{aligned}
$$

commutes in the sense that

$$
N(A) \otimes_{A \times \widehat{S}_{W}}\left({ }_{A} X_{B} \times \widehat{S}_{W}\right) \cong\left({ }_{A} X_{B} \times \widehat{S}_{V} \times{ }_{r} S_{U}\right) \otimes_{B \times \widehat{S}_{V} \times{ }_{r} S_{U}} N(B)
$$

as right-Hilbert $A \times \widehat{S}_{V} \times{ }_{r} S_{U}-B \times \widehat{S}_{W}$ bimodules.

ProOF: By definition we have an imprimitivity bimodule ${ }_{K} X_{B}$, a nondegenerate homomorphism $\psi: A \rightarrow M(K)$, and a coaction $\delta_{K}$ of $S_{V}$ on $K=\mathcal{K}_{B}(X)$ such that $\psi$ is $\delta_{A}-\delta_{K}$ equivariant and $\left(\delta_{K}, \delta_{X}, \delta_{B}\right)$ is an imprimitivity bimodule coaction of $S_{V}$ on ${ }_{K} X_{B}$ which is nondegenerate by assumption. Our strategy will be to prove a version of Diagram 4.1 for the imprimitivity bimodule ${ }_{K} X_{B}$, a version for the standard bimodule ${ }_{A} K_{K}$, and then to combine them using the decomposition Lemma 2.2.

First consider the imprimitivity bimodule ${ }_{K} X_{B}$ : let $L=L(X)$ be the linking algebra of $X$, let $p=\left(\begin{array}{ll}1 & 0 \\ 0 & 0\end{array}\right)$ and $q=\left(\begin{array}{ll}0 & 0 \\ 0 & 1\end{array}\right)$ be the canonical projections in $M(L)$, and let $\delta_{L}$ be the associated coaction of $S_{V}$ on $L$. Then $\delta_{L}$ is injective since $\delta_{K}$ and $\delta_{B}$ (hence also $\delta_{X}$ ) are. Since $\delta_{B}$ is nondegenerate, we have

$$
\begin{aligned}
\overline{\operatorname{span}}\left\{\delta_{X}(X) \cdot(1 \otimes S)\right\} & =\overline{\operatorname{span}}\left\{\delta_{X}(X \cdot B) \cdot(1 \otimes S)\right\} \\
& =\overline{\operatorname{span}}\left\{\delta_{X}(X) \cdot \delta_{B}(B)(1 \otimes S)\right\} \\
& =\overline{\operatorname{span}}\left\{\delta_{X}(X) \cdot(B \otimes S)\right\} \\
& =X \otimes S
\end{aligned}
$$

similarly, the nondegeneracy of $\delta_{K}$ implies that $\overline{\operatorname{span}}\left\{\widehat{\delta_{X}(X)} \cdot(1 \otimes S)\right\}=\widetilde{X \otimes S}$. It follows easily that $\delta_{L}$ is nondegenerate as well. Thus, by [18, Theorem 3.4], we have a $K \times_{\delta_{K}} \widehat{S}_{V} \times_{\hat{\delta}_{K} \mid, r} S_{U}-K \times_{\delta_{K} \mid} \widehat{S}_{W}$ imprimitivity bimodule $N(K)$, and an $L \times_{\delta_{L}} \widehat{S}_{V} \times_{\hat{\delta}_{L} \mid, r} S_{U}$ - $L \times_{\delta_{L} \mid} \widehat{S}_{W}$ imprimitivity bimodule $N(L)$. We claim that

$$
N(K) \otimes_{K \times \widehat{S}_{W}}\left({ }_{K} X_{B} \times \widehat{S}_{W}\right) \cong\left({ }_{K} X_{B} \times \widehat{S}_{V} \times{ }_{r} S_{U}\right) \otimes_{B \times \widehat{S}_{V} \times S_{V}} N(B)
$$

as $K \times \widehat{S}_{V} \times_{r} S_{U}-B \times \widehat{S}_{W}$ imprimitivity bimodules.

$$
\begin{aligned}
\text { Now } \delta_{L} \mid=\left(\begin{array}{ll}
\delta_{K} \mid & \delta_{X} \mid \\
\delta_{\tilde{X}} \mid & \delta_{B} \mid
\end{array}\right), & \text {,o } \\
& L(X) \times_{\delta_{L} \mid} \widehat{S}_{W} \cong L\left(X \times_{\delta_{x} \mid} \widehat{S}_{W}\right) .
\end{aligned}
$$


Also, $\left(L(X) \times \widehat{S}_{V},\left(\widehat{S}_{V}\right)_{p}, \widehat{\delta}_{L}\right) \cong\left(L\left(X \times \widehat{S}_{V}\right),\left(\widehat{S}_{V}\right)_{p}, \varepsilon_{L}\right)$, where $\varepsilon_{L}=\left(\begin{array}{ll}\widehat{\delta}_{K} & \widehat{\delta}_{X} \\ \widehat{\delta}_{\tilde{X}} & \widehat{\delta}_{B}\end{array}\right)$. It follows that the coactions $\widehat{\delta}_{L} \mid$ and $\varepsilon_{L} \mid=\left(\begin{array}{ll}\widehat{\delta}_{K} \mid & \widehat{\delta}_{X} \mid \\ \hat{\delta}_{\tilde{X} \mid} & \widehat{\delta}_{B} \mid\end{array}\right)$ of $\left(\widehat{S}_{U}\right)_{p}$ are isomorphic, and therefore that their reductions are, so that

$$
L(X) \times_{\delta_{L}} \widehat{S}_{V} \times_{\hat{\delta}_{L} \mid, r} S_{U} \cong L\left(X \times_{\delta_{X}} \widehat{S}_{V}\right) \times_{\varepsilon_{L} \mid, r} S_{U} \cong L\left(X \times_{\delta_{X}} \widehat{S}_{V} \times_{\tilde{\delta}_{x} \mid, r} S_{U}\right)
$$

Let $p_{W}, q_{W} \in M\left(L\left(X \times \widehat{S}_{W}\right)\right)$ and $p_{U}, q_{U} \in M\left(L\left(X \times \widehat{S}_{V} \times_{r} S_{U}\right)\right)$ be the canonical projections. Then using Equations (4.3) and (4.4) to view $N(L)$ as an $L\left(X \times \widehat{S}_{V} \times_{r} S_{U}\right)$ - $L\left(X \times \widehat{S}_{W}\right)$ imprimitivity bimodule, [7, Lemma 4.6] gives us a $K \times \widehat{S}_{V} \times_{\mathrm{r}} S_{U}-B \times \widehat{S}_{W}$ imprimitivity bimodule isomorphism

$$
\begin{aligned}
\left(p_{U} \cdot N(L) \cdot p_{W}\right) & \otimes_{K \times \widehat{S}_{W}}\left({ }_{K} X_{B} \times \widehat{S}_{W}\right) \\
& \cong\left({ }_{K} X_{B} \times \widehat{S}_{V} \times{ }_{r} S_{U}\right) \otimes_{B \times \widehat{S}_{V} \times_{r} S_{U}}\left(q_{U} \cdot N(L) \cdot q_{W}\right) .
\end{aligned}
$$

Thus, in order to establish Equation (4.2) we only need imprimitivity bimodule isomorphisms $p_{U} \cdot N(L) \cdot p_{W} \cong N(K)$ and $q_{U} \cdot N(L) \cdot q_{W} \cong N(B)$, and by symmetry it suffices to prove the first. Now the isomorphism $L\left(X \times \widehat{S}_{V} \times_{r} S_{U}\right) \cong L\left(X \times \widehat{S}_{V}\right) \times_{r} S_{U}$ takes $p_{U}$ to $\varepsilon_{L}\left(p_{V}\right)$, and the isomorphisms $L\left(X \times \widehat{S}_{W} \cong L(X) \times \widehat{S}_{W} \cong\left(L(X) \times \widehat{S}_{V}\right)^{\hat{\delta}_{L} \mid} \cong\right.$ $L\left(X \times \widehat{S}_{V}\right)^{\varepsilon_{L} \mid}$ carry $p_{W}$ to $p_{V}$. Therefore, Lemma 3.2 (applied to the coaction of $S_{\hat{U}}$ on $L \times_{\mathrm{r}} \widehat{S}_{V}$ equivalent to $\left.\widehat{\delta}_{L} \mid\right)$ tells us that

$$
p_{U} \cdot N(L) \cdot p_{W} \cong \varepsilon_{L}\left(p_{V}\right) \cdot \mathcal{F}\left(L \times_{r} \widehat{S}_{V}\right) \cdot p_{V} \cong \mathcal{F}\left(K \times_{r} \widehat{S}_{V}\right) \cong N(K),
$$

which gives Equation (4.2).

Next we consider the standard bimodule ${ }_{A} K_{K}$ with the right-Hilbert bimodule coaction $\left(\delta_{A}, \delta_{K}, \delta_{K}\right)$. We claim that

$$
N(A) \otimes_{A \times \hat{S}_{W}}\left({ }_{A} K_{K} \times \widehat{S}_{W}\right) \cong\left({ }_{A} K_{K} \times \widehat{S}_{V} \times{ }_{r} S_{U}\right) \otimes_{K \times \hat{S}_{V} \times_{r} S_{U}} N(K)
$$

as right-Hilbert $A \times \widehat{S}_{V} \times_{r} S_{U}-K \times \widehat{S}_{W}$ bimodules; by [12, Lemma 5.3] and Lemma 2.1, it is enough to show that there is a nondegenerate imprimitivity bimodule homomorphism $\Psi$ from $N(A)$ to $M(N(K))$ with coefficient maps $\psi \times \widehat{S}_{V} \times_{r} S_{U}$ and $\psi \times \widehat{S}_{W}$. Applying Lemma 3.3 to the nondegenerate homomorphism $\psi \times \widehat{S}_{V}: A \times \widehat{S}_{V} \rightarrow M\left(K \times \widehat{S}_{V}\right)$, which is equivariant for the coactions (of $S_{\widehat{U}}$ equivalent to) $\widehat{\delta}_{A} \mid$ and $\widehat{\delta}_{K} \mid$ of $\widehat{S}_{U}$, we obtain a nondegenerate imprimitivity bimodule homomorphism $\Psi: \mathcal{F}\left(A \times \widehat{S}_{V}\right) \rightarrow M\left(\mathcal{F}\left(K \times \widehat{S}_{V}\right)\right)$ with coefficient maps $\psi \times \widehat{S}_{V} \times_{r} S_{U}$ and $\left.\left(\psi \times \widehat{S}_{V}\right)\right|_{\left(A \times \widehat{S}_{V}\right)^{\beta_{A}}}$. Now by definition, $\psi \times \widehat{S}_{V}=$ $\left(j_{K}^{V} \circ \psi\right) \times \mu_{K}^{V}$, and Ng's isomorphism $\phi_{A}: A \times \widehat{S}_{W} \rightarrow\left(A \times \widehat{S}_{V}\right)^{\hat{\delta}_{A} l}$ is $j_{A}^{V} \times\left(\mu_{A}^{V} \circ \rho_{W, V}\right)$. Thus,

$$
\begin{aligned}
\phi_{K} \circ\left(\psi \times \widehat{S}_{W}\right) & =\left(j_{K}^{V} \times\left(\mu_{K}^{V} \circ \rho_{W, v}\right)\right) \circ\left(\left(j_{K}^{W} \circ \psi\right) \times \mu_{K}^{W}\right) \\
& =\left(j_{K}^{V} \circ \psi\right) \times\left(\mu_{K}^{V} \circ \rho_{W, V}\right) \\
& =\left(\left(j_{K}^{V} \circ \psi\right) \times \mu_{K}^{V}\right) \times\left(j_{A}^{V} \times\left(\mu_{A}^{V} \circ \rho_{W, V}\right)\right) \\
& =\left(\psi \times \widehat{S}_{V}\right) \circ \phi_{A} .
\end{aligned}
$$


This shows that the isomorphisms $\phi_{A}: A \times \widehat{S}_{W} \rightarrow\left(A \times \widehat{S}_{V}\right)^{\widehat{\delta}_{A} \mid}$ and $\phi_{K}: K \times \widehat{S}_{W} \rightarrow$ $\left(K \times \widehat{S}_{V}\right)^{\hat{\delta}_{K} \mid}$ carry the coefficient map $\left.\left(\psi \times \widehat{S}_{V}\right)\right|_{\left(A \times \widehat{S}_{V}\right)^{\hat{\delta}_{A}}}$ to $\psi \times \widehat{S}_{W}$; in other words, viewed as a map of $N(A)$ into $M(N(K)), \Psi$ is a nondegenerate imprimitivity bimodule homomorphism with coefficient maps $\psi \times \widehat{S}_{V} \times S_{U}$ and $\psi \times \widehat{S}_{W}$, which establishes Equation (4.5).

We now have a prism

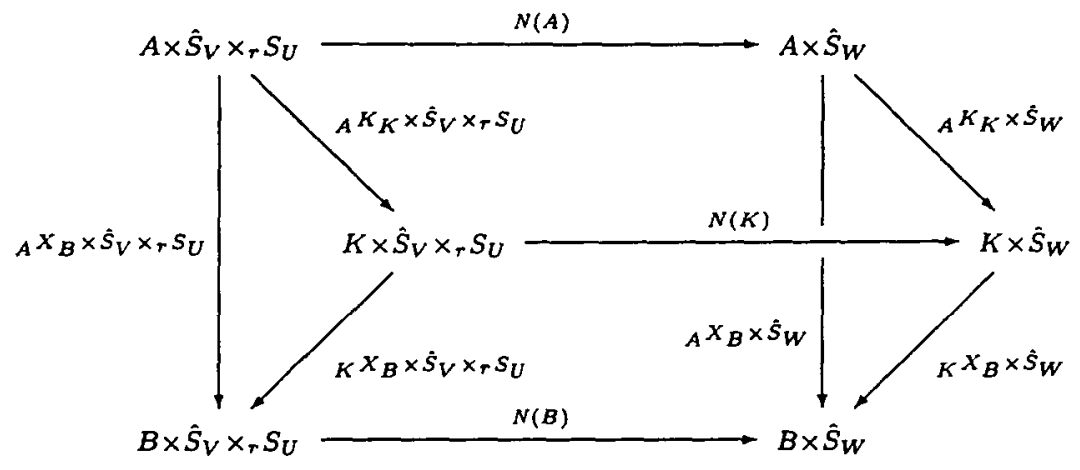

in which the front two faces commute by the above arguments, and the commutativity of the back face is the desired result; it only remains to show that the two side triangles commute. That is, we need to know that

$$
{ }_{A} X_{B} \times \widehat{S}_{V} \times{ }_{r} S_{U} \cong\left({ }_{A} K_{K} \times \widehat{S}_{V} \times{ }_{r} S_{U}\right) \otimes_{K \times \widehat{S}_{V} \times S_{V}}\left({ }_{K} X_{B} \times \widehat{S}_{V} \times{ }_{r} S_{U}\right)
$$

and

$$
{ }_{A} X_{B} \times \widehat{S}_{W} \cong\left({ }_{A} K_{K} \times \widehat{S}_{W}\right) \otimes_{K \times \widehat{S}_{W}}\left({ }_{K} X_{B} \times \widehat{S}_{W}\right)
$$

as right-Hilbert $A \times \widehat{S}_{V} \times_{r} S_{U}-B \times \widehat{S}_{V} \times_{r} S_{U}$ and $A \times \widehat{S}_{W}-B \times \widehat{S}_{W}$ bimodules, respectively. But this follows from Lemma 2.2, in the first case applied to the coaction $\left(\widehat{\delta}_{A}\left|, \widehat{\delta}_{X}\right|, \widehat{\delta}_{B} \mid\right)$ of $\widehat{S}_{U}$ on ${ }_{A} X_{B} \times \widehat{S}_{V}$ and then using $K \times \widehat{S}_{V} \cong{ }_{A} K_{K} \times \widehat{S}_{V}$ from Lemma 2.1.

REMARK 4.2. For an imprimitivity bimodule coaction $\left(\delta_{K}, \delta_{X}, \delta_{B}\right)$ of a Hopf $C^{*}$ algebra, it is probably true that $\delta_{K}$ is nondegenerate whenever $\delta_{B}$ is; this would simplify the hypotheses of Theorem 4.1 somewhat. Unfortunately, we have been unable to find a proof. (For group coactions, it is true - see [11, Proposition 2.3] - and the proof is fairly nontrivial.) It may be possible to finesse the problem, but since our main point here is to illustrate our approach to imprimitivity theorems, we have chosen not to get mired in nondegeneracy issues.

\section{REFERENCES}

[1] S. Baaj and G. Skandalis, ' $C$ *algèbres de Hopf et théorie de Kasparov équivariante', $K$-Theory 2 (1989), 683-721. 
[2] S. Baaj and G. Skandalis, 'Unitaires multiplicatifs et dualité pour les produits croisés de $C^{*}$-algebres', Ann. Sci. École Norm. Sup. 26 (1993), 425-488.

[3] S. Echterhoff, 'Duality of induction and restriction for abelian twisted covariant systems', Math. Proc. Camb. Philos. Soc. 116 (1994), 301-315.

[4] S. Echterhoff, S. Kaliszewski, J. Quigg and I. Raeburn, 'Imprimitivity theorems as natural equivalences', (in preparation, 2000).

[5] S. Echterhoff, S. Kaliszewski and I. Raeburn, 'Crossed products by dual coactions of groups and homogeneous spaces', J. Operator Theory 39 (1998), 151-176.

[6] S. Echterhoff and I. Raeburn, 'Multipliers of imprimitivity bimodules and Morita equivalence of crossed products', Math. Scand. 76 (1995), 289-309.

[7] S. Echterhoff and I. Raeburn, 'The stabilisation trick for coactions', J. reine angew. Math. 470 (1996), 181-215.

[8] E. Gootman and A. Lazar, 'Applications of non-commutative duality to crossed product $C^{*}$-algebras determined by an action or coaction', Proc. London Math. Soc. 59 (1989), 593-624.

[9] P. Green, 'The local structure of twisted covariance algebras', Acta Math. 140 (1978), 191-250.

[10] S. Imai and H. Takai, 'On a duality for $C^{*}$-crossed products by a locally compact group', J. Math. Soc. Japan 30 (1978), 495-504.

[11] S. Kaliszewski and J. Quigg, 'Imprimitivity for $C^{*}$-coactions of non-amenable groups', Math. Proc. Cambridge Philos. Soc. 123 (1998), 101-118.

[12] S. Kaliszewski, J. Quigg and I. Raeburn, 'Duality of restriction and induction for $C^{*}$-coactions', Thans. Amer. Math. Soc. 349 (1997), 2085-2113.

[13] Y. Katayama, 'Takesaki's duality for a non-degenerate co-action', Math. Scand. 55 (1985), 141-151.

[14] E.C. Lance, Hilbert $C^{*}$-modules, London Math. Soc. Lecture Note Ser. 210 (Cambridge University Press, Cambridge, 1995).

[15] K. Mansfield, 'Induced representations of crossed products by coactions', J. Funct. Anal. 97 (1991), 112-161.

[16] C.K. Ng, 'Coactions and crossed products of Hopf $C^{*}$-algebras II: Hilbert $C^{*}$-modules', (preprint, 1995).

[17] C.K. Ng, 'Coactions and crossed products of Hopf $C^{*}$-algebras', Proc. London Math. Soc. 72 (1996), 638-656.

[18] C.K. Ng, 'Morita equivalences between fixed point algebras and crossed products', Proc. Cambridge Philos. Soc. 125 (1999), 43-52.

[19] C.K. Ng, 'Morphisms of multiplicative unitaries', J. Operator Theory 38 (1997), 203-224.

[20] M. Nilsen, 'Duality for crossed products of $C^{*}$-algebras by non-amenable groups', Proc. Amer. Math. Soc. 126 (1998), 2969-2978.

[21] M.A. Rieffel, 'Induced representations of $C^{*}$-algebras', Adv. Math. 13 (1974), 176-257.

[22] Y. Watatani, 'Index for $C^{*}$-subalgebras', Mem. Amer. Math. Soc. 83 (1990), pp. 117. 
Department of Mathematics

Arizona State University

Tempe AZ 85287

United States of America

e-mail: kaz@math.la.asu.edu

quigg@math.la.asu.edu 